\title{
Mitteilungen
}

\section{Ausschreibung: Preis der Mogens und Wilhelm Ellermann-Stiftung}

Die Schweizerische Neurologische Gesellschaft verleiht 2020 zum siebzehnten Mal den Preis der Mogens und Wilhelm Ellermann-Stiftung im Betrage von $20000 \mathrm{CHF}$.

Der Preis wird vergeben

a) für eine wissenschaftliche Arbeit aus dem Gebiet der neurologischen Wissenschaften, die in den letzten zwei Jahren publiziert oder von einer international anerkannten Zeitschrift zur Publikation angenommen worden ist;

b) für eine Monographie, die nicht den Charakter eines Lehrbuches oder einer Übersicht hat;

c) für ein wissenschaftliches Gesamtwerk aus dem Gebiet der neurologischen Wissenschaften.
Die Arbeit muss von einem oder mehreren Schweizer Wissenschaftlern oder von Ausländern, die an einer schweizerischen Klinik oder einem schweizerischen Institut tätig sind, verfasst worden sein. Vorzugsweise sollen jüngere Autoren, die nicht oder erst kürzlich habilitiert worden sind, berücksichtigt werden.

Bewerbungen mit Curriculum Vitae und Publikationsliste (nur peer-reviewed Originalarbeiten) sind bis zum 31. Januar 2020 zu adressieren an Prof. Dr. med. C. L. Bassetti, Direktor und Chefarzt, Universitätsklinik für Neurologie, Inselspital Bern, 3010 Bern. Bitte Bewerbung per E-Mail einreichen an: irene.kaufmann[at] insel.ch 